\title{
BMJ Open Protocol for a multicentre, prospective cohort study of clinical, proteomic and genomic patterns associated with osteoporosis to develop a multidimensional fracture assessment tool: the PoCOsteo Study
}

\author{
Patricia Khashayar, ${ }^{1}$ Hans Peter Dimai, ${ }^{2}$ Nahid Moradi, ${ }^{3}$ Noushin Fahimfar, ${ }^{3}$ \\ Safoora Gharibzadeh, ${ }^{4}$ Afshin Ostovar, ${ }^{3}$ Iraj Nabipour, ${ }^{5}$ Bagher Larijani (1) ${ }^{6}$
}

To cite: Khashayar P, Dimai HP, Moradi N, et al. Protocol for a multicentre, prospective cohort study of clinical, proteomic and genomic patterns associated with osteoporosis to develop a multidimensional fracture assessment tool: the PoCOsteo Study. BMJ Open 2020;10:e035363. doi:10.1136/ bmjopen-2019-035363

- Prepublication history for this paper is available online. To view these files, please visit the journal online (http://dx.doi. org/10.1136/bmjopen-2019035363).

Received 29 0ctober 2019 Revised 12 July 2020 Accepted 22 July 2020
Check for updates

(C) Author(s) (or their employer(s)) 2020. Re-use permitted under CC BY-NC. No commercial re-use. See rights and permissions. Published by BMJ.

For numbered affiliations see end of article.

Correspondence to Dr Bagher Larijani; larijanib@tums.ac.ir

\section{ABSTRACT}

Introduction The HORIZON 2020 project PoCOsteo aims (1) to develop a multidimensional fracture risk assessment tool which would take into account all factors known to be related to an individual's fracture risk. The fracture risk model is intended to be developed in two different populations, namely a European and a Middle Eastern one; (2) to develop a medical device, which would measure and/or quantify proteomic as well as genomic factors as present in whole blood samples collected through finger prick; (3) to test the clinical applicability and the validity of prototypes of the to be developed point of care device at both clinical centres.

Methods and analysis This article presents the protocol of this prospective cohort that will be carried out independently at two different centres (Division of Endocrinology and Diabetology at the Medical University of Graz (MUG) as a clinic-based cohort, and Endocrinology and Metabolism Research Institute (EMRI) at the Tehran University of Medical Sciences (TUMS) as a population-based cohort). The final aim is to develop a fracture risk assessment model, which would include clinical risk factors, biochemical markers of bone turnover, as well as specific genomic factors. The derivation cohorts will consist of individuals aged 50 years and above. The period of observation for each patient will be 12 months; an extension phase, which would last for another 2 years, is also planned.

Ethics and dissemination These studies are conducted in accordance with the World Medical Association Declaration of Helsinki. The Iranian part was approved by the Research Ethics Committee of EMRI, TUMS. The Austrian part was approved by the Ethics Committee of the Medical University of Graz. Based on the gathered information, a multidimensional fracture assessment tool will be designed which will later be added to the PoCOsteo device.

\section{INTRODUCTION}

Background and rationale

The world population is ageing rapidly. The proportion of individuals aged over 60 years will double, from about $11 \%$ to $22 \%$ between 2000 and 2050, suggesting that about 2 billion
Strengths and limitations of this study

- The multicentric cohort design of the study will allow the recruitment of a large group of diverse individuals. This will improve not only the knowledge on osteoporosis and osteoporotic fracture but also the validity and generalisability of the to be developed multidimensional fracture assessment tool. In other words, this is one of the few studies to be conducted on a European and Middle Eastern population using a nearly similar protocol. And therefore, provides a unique opportunity for the investigators to compare their findings in the two studied populations.

- Moreover, nowadays, no fracture risk assessment tool based on fracture risk-related proteomic, genomic and clinical factors exists to our knowledge. The modelling to be developed in this study will be the first in this regard.

- The additional amount of blood stored in the biobanks during the research will provide an exceptional chance to study other factors yet to be determined in future projects.

- The difference in the nature of the study populations between the two centres could result in some disparities in the results. As the subjects are collected from a single centre in each continent, the generalisability of the results to the whole European and Middle Eastern population might not be possible. As the study is conducted on the elderly population, lost to follow-up due to underlying health conditions and death is inevitable.

individuals are expected to be over 60 years in $2050{ }^{1}$

The rise in the number of the elderly highlights the surging need for understanding the impact and consequences of ageing as well as the necessity to research into the prevention 
and management strategies for non-communicable diseases (NCDs).

To achieve these goals, research into the health problems of the elderly and preserving their quality of life and independence has become a top priority in many health settings. ${ }^{2}$ Physical disability and cognitive impairment are the two main risks that threaten the independence of the elderly. The former is commonly caused by musculoskeletal disorders such as osteoporosis. ${ }^{3-6}$

Osteoporosis is frequently referred to as a 'silent disease,' indicating that the development of the disease may remain unrecognised until the first fracture occurs. ${ }^{7}$ Osteoporosis and related fractures impose a heavy socioeconomic burden and are thus considered as a major health concern in many countries worldwide. So far, no easily accessible and widely available methods with sufficient sensitivity for early detection of osteoporosis have been developed.

In 2010, 22 million women and 5.5 million men were estimated to have osteoporosis in the EU. In the same year, about 3.5 million new fragility fractures were sustained in different member states. ${ }^{8}$ These fractures accounted for 1180000 quality-adjusted life-years (QALY) lost and were estimated to impose a burden of about $€ 37$ billion. In 2025, these costs are expected to increase by $25 \%$. Iran, as well, is affected by this phenomenon with the disease depriving Iranians of 36'026 healthy years of life (18 757 in men and 17270 in women) in 2001..$^{910}$

Currently, the gold-standard and the validated method to investigate osteoporosis is dual energy X-ray absorptiometry (DXA), which is usually available in tertiary or imaging centres. While there is good evidence that low bone mineral density (BMD) is associated with an increase in fracture risk, the sensitivity of this method to detect at-risk individuals is below $50 \%$ and more than one-third of fractures occur in individuals with normal BMD. ${ }^{11}$ Moreover, improvements in bone density appear in DXA after 2 years, which devalues DXA as a monitoring tool. On the other hand, the test is expensive with a range of $€ 48$ to some $€ 300$. The annual costs from a screening visit to the full investigation are calculated to round up at around $€ 44000-€ 88000 /$ QALY. $^{12}$ This figure highlights the need for better vetting of the patients who are referred for imaging to maximise the cost-effectiveness while protecting the patients against unnecessary exposure to X-ray radiation.

Bone turnover biomarkers (BTMs), such as osteocalcin (OC), C-terminal telopeptide of type I procollagen (CTx), N-propeptide of type I procollagen (PINP), bonespecific alkaline phosphatase (bALP) or tartrate-resistant acid phosphatase (TRAP), on the other hand, provide useful information on the momentary balance between bone formation and resorption and thus fracture risk, particularly in postmenopausal women. ${ }^{13}$ However, utilisation of these markers is very limited due to their preanalytical and analytical variability along with the need for expensive and sophisticated laboratory equipment, such as ELISA or electrochemiluminescence-immunoassay (ECLIA).

In recent years, genetic variability has also been shown to be related to an individual's fracture risk and/or BMD values; as a result, the measurement of parameters such as single-nucleotide polymorphisms (SNPs) and micro RNAs are becoming more important. This is while their measurement is limited to few and highly specialised centres as not only it requires highly specialised personnel, but even more sophisticated and more expensive laboratory equipment compared with the biochemical markers.

Apart from these variables, clinical risk factors (CRFs) play a pivotal role in predicting an individual's absolute fracture probability. As a result, CRFs have been integrated into a variety of fracture risk assessment tools such as FRAX and QFracture. ${ }^{14}{ }^{15}$ Nevertheless, no fracture risk assessment tool taking into account proteomic and genetic factors is currently available.

The PoCOsteo project is an EU-funded H2020 project, designed to achieve this goal by providing the physicians with a tool to perform an individual risk assessment for each patient while bringing together biomarker measurement, profiling of genetic variations and assessing the underlying risk factors. This approach will enhance the predictive accuracy of fracture risk and provide the affected individuals with personalised care even in rural areas, where access to current state-of-the-art equipment is limited. This real-time measurement device will also revolutionise the treatment monitoring process, making it more efficient while reducing the low-compliance rate commonly reported in osteoporotic patients. ${ }^{16}$ The International Osteoporosis Foundation (IOF), an international non-governmental organisation with the mission to advance the understanding of osteoporosis and to promote prevention, diagnosis and treatment of the disease worldwide, supports the project.

\section{Specific aims}

The overall aim of this project is to develop a multidimensional fracture risk assessment model and validate it in practice. In brief, the device will measure both BTMs and genetic factors using a finger prick blood sample. ${ }^{17}$ It then integrates the respective results into an algorithm, which also accounts for CRFs to estimate an individual's risk of developing a fracture.

To detect possible population-specific differences, data derivation cohorts are recruited in parallel but independently at two different study centres, namely the Medical University of Graz, representing a European population, and the Endocrinology and Metabolism Research Institute (EMRI), Tehran University of Medical Sciences (TUMS), representing a Middle-Eastern population.

The clinical studies also aim at investigating the prevalence and incidence of osteoporosis and osteoporotic fracture as well as their risk factors in the elderly populations. They also assess possible preanalytical variability in BTM measurement. 


\begin{tabular}{|c|c|c|c|}
\hline Baseline & Year 1 & $\begin{array}{l}\text { Year } 2 \text { (optional; } \\
\text { "extension Phase") }\end{array}$ & $\begin{array}{l}\text { Year } 3 \text { (optional; } \\
\text { "extension Phase") }\end{array}$ \\
\hline$\downarrow$ & * & $\downarrow$ & $\downarrow$ \\
\hline History (CRFs) & Histary (CRFs) & History (CRFs) & History (CRFs) \\
\hline Physical Examination (Iran only) & Physical Examination (Iran only) & Physical Examination (Iran only) & Physical Examination (Iran only) \\
\hline$B M D+V F A$ & $B M D+V F A$ & $B M D+V F A$ & $B M D+V F A$ \\
\hline HR-pQCT (subgroup Graz only) & HR-pQCT (subgroup Graz only) & HR-pQCT (subgroup Graz only) & HR-pQCT (subgroup Graz only) \\
\hline Routine Parameters & Routine Parameters & Routine Parameters & Routine Parameters \\
\hline Hormonal Status (optional) & Hormonal Status (optional) & Hormonal Status (optional) & Hormonal Status (optional) \\
\hline Bone-Specific lab & Bone-Specific lab & Bone-Specific lab & Bone-Specific lab \\
\hline $\begin{array}{l}\text { Additional Tests (FGF 23, } \\
\text { myostin, Sclerostin) (optional Graz) }\end{array}$ & $\begin{array}{l}\text { Additional Tests (FGF 23, } \\
\text { myostin, Sclerostin) (optional Graz) }\end{array}$ & $\begin{array}{l}\text { Additional Tests (FGF 23, } \\
\text { myostin, Sclerostin) (optional Graz) }\end{array}$ & $\begin{array}{l}\text { Additional Tests (FGF } 23 \text {, } \\
\text { myostin, Sclerostin) (optional Graz) }\end{array}$ \\
\hline $\begin{array}{l}\text { Samples for freezer (aliquots for } \\
\text { genomic/proteomic tests; SNPs, miRNAs) }\end{array}$ & $\begin{array}{l}\text { Samples for freezer (aliquots for } \\
\text { genomic/proteomic tests; SNPs, miRNAs) }\end{array}$ & $\begin{array}{l}\text { Samples for freezer (aliquots for } \\
\text { genomic/proteomic tests; SMPs, miRNAs) }\end{array}$ & $\begin{array}{l}\text { Samples for freezer (aliquots for } \\
\text { genomic/proteomic tests; SNPs, miRNAs) }\end{array}$ \\
\hline $\begin{array}{l}\text { Samples for biobank (aliquots for } \\
\text { genomic/proteomic tests; } 5 \mathrm{NPs} \text {, miRNAs) }\end{array}$ & $\begin{array}{l}\text { Samples for biobank (aliquots for } \\
\text { genomic/proteomic tests; SNPs, miRNAs) }\end{array}$ & $\begin{array}{l}\text { Samples for biobank (aliquots for } \\
\text { genomic/proteomic tests; SNPs, miRNAs) }\end{array}$ & $\begin{array}{l}\text { Samples for biobank (aliquots for } \\
\text { genomic/proteomic tests; SNPs, miRNAs) }\end{array}$ \\
\hline
\end{tabular}

Figure 1 Tasks to be completed at each visit during the BEH stage two study and Graz. CRFs, clinical risk factors; BEH, Bushehr elderly health; BMD, bone mineral density; HR-pQCT, high-resolution peripheral CT; miRNAs, micro RNS; SNPs, singlenucleotide polymorphisms; VFA, vertebral fracture assessment.

These clinical studies also provide the opportunity for the PoCOsteo device to be tested in a clinical environment to determine its diagnostic accuracy in measuring the BTM levels and detecting selected genes in comparison with ECLIA and PCR, respectively. They also help with calculating the diagnostic value of the PoCOsteo device in predicting individual fracture risk and monitoring osteoporosis treatment in comparison to DXA.

\section{STUDY DESIGN}

As mentioned earlier, this prospective multicentric cohort study is conducted in two different study centres. Despite the differences in the recruited population, the study protocols are congruent so that the final results would be comparable. Using a cohort approach, data for each centre are collected from the beginning of spring 2018 and the subjects will be followed up to 3 years or at least until the end of the project in autumn 2021 (The device will be applied in real time to a subsample of the collected blood samples at Year 3. Moreover, after Year 3, all Iranian participants will be followed according to the Bushehr elderly health (BEH) protocol). A schematic diagram of the study design and the tests to be performed at each visit is illustrated in figure 1 .

\section{Study population}

The target population of this study is recruited from two study sites with two different approaches.

\section{Iran: Bushehr elderly health programme}

$\mathrm{BEH}$ is a prospective cohort study conducted by the EMRI of TUMS and Persian Gulf Marine Biotechnology Research Centre (PGTMRC) with the aim of investigating the prevalence of NCDs in an elderly population. ${ }^{18}$ The current study is designed as an extension to the second stage of BEH and will mainly focus on musculoskeletal diseases (in line with the main objective of PoCOsteo).

Two thousand new subjects are enrolled through the same method used in the previous stage of the BEH programme. In brief, the participants are selected through a multistage, stratified cluster random sampling method. Based on the classifications made by the Bushehr municipality, the city is stratified into 75 strata. Numbers are assigned to the blocks (as clusters) of each stratum and then randomly selected. All eligible people residing in each selected block will be invited until the determined sample size for that stratum is reached. This number is calculated proportionally to the number of households residing in each stratum.

Both male and female participants aged 50 years and over who have resided in Bushehr port for at least 1 year prior the recruitment and have no plan to leave the city for at least the next 5 years and agree to be part of the study will be recruited. Those with a positive history of bilateral hip replacement surgery or not having adequate physical and mental ability to participate in the evaluation programme will be excluded.

The invitations will be presented to all the randomly selected households with inhabitants meeting the age criteria through a door-to-door household visit. They contain instructions on how to contact the research organisation and the responsible staff to participate in the study.

An android application is developed to manage invitations based on national ID codes. The participants who have been invited to participate in the previous stage of BEHP will be removed from the invitation list.

\section{Austria: Graz study}

The Graz study is similarly designed as a prospective cohort study but will be carried out at the outpatient osteoporosis clinic of the Division of Endocrinology and Diabetology in the University Hospital of Graz. All women and men 50 years and above who are referred to the osteoporosis clinic will be asked to participate in this study.

\section{Initial assessment}

The participants from both centres who meet the study inclusion criteria will be informed in more detail about the purpose and the cornerstones of the present study. 
After having obtained a written informed consent from the respective patient, the study nurse will start collecting the baseline data including anthropometric parameters and CRFs known to be associated with fracture risk. A baseline BMD and vertebral fracture assessment (VFA) will be also performed. A subgroup of the Austrian subjects will also be selected to undergo high-resolution peripheral CT (HR-pQCT). Thereafter, blood will be drawn from a cubital vein of each patient.

Based on previous studies, we assume a prevalence of about $30 \%$ for osteoporosis, indicating that approximately 600 individuals will be diagnosed with osteoporosis at baseline. ${ }^{19} 20$ This subpopulation along with those diagnosed with osteoporosis during the study will be referred to a specialist with ample experience in managing osteoporosis. The research nurse will also follow these participants. She/he will also follow the occurrence of main outcomes (mortality, hospital admissions and fractures) for all participants periodically through phone calls.

\section{Follow-up assessments}

The first follow-up visit will take place 12 months after recruitment. Shortly before the end of year 1 , the study nurse will contact the respective patients and invite them to the follow-up visit. In case a patient is unreachable, the study coordinator will be contacted for further action.

During this follow-up, the study nurse will take history and report any relevant information. Venous blood will be drawn (from a cubital vein) and a follow-up BMD and VFA, and, if applicable, a follow-up HR-pQCT will be performed. In case the project consortium decides to extend the clinical study, 2-year and/or 3-year follow-up visits will be conducted.

At the beginning of year 4, the PoCOsteo device will be ready for use and thus the BTM measurements and SNP studies will be performed in real-time using the device in a subsample of the study population (both osteoporotic and non-osteoporotic subjects). The results will be then compared with the respective gold-standard methods (ECLIA for biochemical markers and PCR for genetic markers).

\section{Patient and public involvement}

As mentioned earlier, research on health problems of the elderly and preserving their quality of life and independence has become a top priority in many health settings and thus the present study was designed to address these concerns and to improve the subjects' quality of life. The subjects were not involved in the design of the current study, but the lessons learnt from previous studies, including their preferences, were taken into account while drafting this protocol. The recruited subjects will be presented with the results of their tests and in case further consultation/treatment is needed, additional sessions will be arranged. Moreover, the subjects will also be educated about osteoporosis and its risk factors during these meetings to improve their awareness.

\section{DATA STORAGE AND MANAGEMENT \\ Data collection}

Patient-specific data will be collected and documented in the case report forms at the time of each visit, and within a week after the results of the diagnostic workups become available to entail all necessary variables. The information to be included in the case report forms include:

\section{Clinical risk factors}

Together with the patient's history, CRFs known or suspected to be associated with an individual's absolute risk of osteoporotic fractures (table 1) will be determined and documented. CRFs will include variables, which are an integral part of the FRAX tool, among others.

\section{Biological samples}

Some $20 \mathrm{~mL}$ of venous blood will be drawn. Blood samples will be dissociated into cells and sera on-site within 20 min after collection and analysis will be performed on the same day. This protocol will be closely followed as the time to dissociation influences the results, especially OC levels.

After the initial analysis, the dissociated sera and the whole blood samples will be stored at $-80^{\circ} \mathrm{C}$ in the PGTMRC biobank (Bushehr, Iran) before being transferred to the EMRI biobank (Tehran, Iran) for further analysis and storage at the same conditions. As for Graz, Austria, the samples will all be stored in the Graz University biobank.

In both centres, blood samples will be collected in the morning after $8-12$ hours of fasting. About $16 \mathrm{~mL}$ of the collected blood will be stored in an evacuated tube. Nine $\mathrm{mL}$ will be stored in three evacuated tubes with Ethylenediaminetetraacetic acid (EDTA) for complete blood count (CBC) analysis, Hemoglobin A1C (HbA1C) measurement, and dissociation of plasma, respectively. The clot will be divided into 10 parts, 6 of which will be used for the tests mentioned in table 2 and the remaining four will be stored in the biobank. Two cryovials of plasma and three cryovials of whole blood along with an extra Pax tube will be stored in the biobank as well.

The BTM measurements will be done in both centres using ECLIA (Roche, Cobas) and similar kits. The genetic variability will be assessed by measuring the presence of specific SNPs in the blood samples using PCR.

\section{Imaging}

- BMD measurement is performed using GE Lunar iDXA for the Austrian study and Hologic DXA (DXA Discovery WI, Hologic, Bedford, Virginia, USA) for the Iranian site. To have comparable results, similar phantoms will be used at both sites to calibrate the devices.

- VFA to be implemented on the DXA machines.

- HR-pQCT (SCANCO Xtreme CT, II Generation) of the lower limb will be performed in a small subgroup of the Austrian patients $(n=50 ; 25$ with prevalent osteoporotic fracture and 25 as controls).$^{21}$ 
Table 1 Information to be collected in clinical studies through CRFs, physical examination and para clinics

CRF Interview Age, gender, smoking status (amount and duration), alcohol consumption status (amount, type and duration), history of fracture (low impact/due to trauma and site), secondary causes of osteoporosis (due to diseases impacting bone health (Chronic Obstructive Pulmonary Disease (COPD), Epilepsy, Dementia, Parkinson's disease, Malignancies, systemic lupus erythematosus, Rheumatoid arthritis, chronic kidney disease diabetes, ...), history of fall, and/or medication affecting bone health (Parkinson medication, dementia medication, antidepressants, anticonvulsants ...)), parental history of hip fracture, glucocorticoid treatment ( $>3$ months and $>5$ $\mathrm{mg}$ prednisolone equivalent). (As for the Iranian part, considering the main objectives of the $\mathrm{BEH}$, cardiovascular risk factors and cognitive function will also be studied. Information on the subjects' socioeconomic status, illicit drug abuse status, physical activity, reproductive information, and duration and amount of sun exposure per day, history of hospital admissions in the previous year (cause and duration), cognitive function and dietary assessment will be collected).

Questionnaire Activities of daily living (ADL) and instrumental ADL, Patient Health Questionnaire-9, health-related quality of life (EuroQoL-5D (EQ-5D)), Short Physical Performance Battery, and Minimal Mental State Examination questionnaires, as well as 6-item Cognitive Impairment Test (As for the Austrian subjects, the Short Form Health Survey-12 (SF-12) Quality of Life questionnaire will be filled out).

Physical examination Anthropometric measurement (weight, height, length of the leg and right arm, and neck, hip, waist, right arm, right femur circumference), blood pressure, the muscular mass, and force evaluation, and evaluation of gait and stance (capability to lift 10-pound weight, capability to walk, capability to get up from a chair/bed, capability to climb a 10-step staircase, time to walk $4.75 \mathrm{~m}$ in seconds, time to stand up from sitting position in seconds, time to walk $2.44 \mathrm{~m}$ in seconds, feet together stance, semi/full tandem stance for $10 \mathrm{~s}$ ).

BEH, Bushehr elderly health; CRFs, clinical risk factors.

Data Handling and storage

All data-related issues will be handled according to the current version of the General Data Protection Regulation. Participants will be given a unique code to protect their identity in any future utilisation of the database. These codes will be used in labelling the case report forms and the digital spreadsheet database as well as all collected blood samples and dissociated sera to ensure anonymity. Only specified research team members can access these data.

To ensure minimal data loss, case report forms will be completed during each visit. As for the Iranian part, interim analysis to investigate participation and dropout rate as well as outlier detection, will be conducted every 3 months to allow timely corrective interventions to prevent data loss.

\section{OUTCOMES AND ANTICIPATED RESULTS}

Prevalent non-vertebral and vertebral fractures

The primary outcome of interest is to determine the number of non-vertebral and vertebral fractures in each population. This is done during history taking.

The age-specific hip fracture incidence in the Austrian $50+$ female and male population is about 650/and $250 / 100000$, respectively. ${ }^{22}$ It can, thus, be anticipated that about 10 hip fractures ( 7 in women and 3 in men) occur within 1 year in the 1000 participants of the current study. As for Iran, the annual incidence of hip fracture per 100000 person-years is 115.2 (95\% CI 107.2 to 123.7) in men and 115.6 (95\% CI 107.4 to 124.3$)$ in women. ${ }^{23}$

It should be noted that these estimates might be an overestimation mainly in the Austrian arm because most of the patients referred to the study centre are already

Table 2 Tests to be performed in each centre

Routine parameters

Bone-specific biomarkers

Hormones (optional-depending

on indication)
Full blood count, fasting blood sugar, $\mathrm{HbA1c}$, renal parameters, hepatic parameters, $\mathrm{C}$ reactive protein, protein electrophoresis, electrolytes including magnesium, lipid profile including high-/low-density lipoprotein -cholesterol

Osteocalcin; carboxyl-terminal telopeptide of type I collagen; bone-specific alkaline phosphatase; tartrate-resistant acid phosphatase; N-propeptide of type I collagen; 25-hydroxyvitamin D; Intact parathyroid hormone

Follicle-stimulating hormone, Luteinising hormone, estradiol, testosterone

Single-nucleotide polymorphisms

Others (These parameters are only Fibroblast Growth Factor 23(FGF 23), Myostatin, Sclerostin

tested in Austria optionally and

depending on the coverage of

thecost) 
suspected of having osteoporosis. On the other hand, this number could also be an underestimation because some of the patients referred to the study centre might have already received treatment for osteoporosis. It should be noted that the total number of fracture observed within 1 year is largely dependent on the proportion of male and female study participants.

As many of the patients will not be able to provide reliable information on the exact fracture location and/or type, verification will be performed by requesting the original patient report and/or the original radiograph. Furthermore, the exact date of the fracture will be determined, as it affects future fracture risk, which would be highest within the first year after a fracture.

Strong epidemiological evidence indicates that the majority of osteoporotic fractures occur at the thoracic and the lumbar spine. However, only around one-third of these fractures come to clinical attention. ${ }^{24}$ It has also been estimated that about seventy per cent of all vertebral fractures remain undetected if no imaging of the spine is performed. To obtain reliable baseline data regarding prevalent vertebral fractures, VFA will be used. ${ }^{25}$ The already available thoracic and lumbar spine radiographs will be accepted for data entry if the radiographs have been taken within the past 6 months before recruitment.

\section{Bone mass}

Identifying osteoporotic patients through measuring their bone mass is the second outcome of interest. BMD measurement is widely recommended by the respective scientific societies worldwide as part of the baseline examinations if osteoporosis is suspected. ${ }^{26}$ As mentioned earlier, DXA is the method of choice and so all patients will undergo BMD measurement of the lumbar spine and the hip. ${ }^{26}$ HR-pQCT will be performed for a subgroup of Austrian subjects to provide more accurate information on their bone structural parameters.

\section{Proteomic parameters}

BTMs have been shown to provide relevant information on the extent of bone resorption and bone formation. ${ }^{27}$ In a position paper published by the IOF, the biomarker taskforce has recommended the use of CTx and PINP as reference markers for all clinical studies, adding one of these markers is sufficient for daily clinical practice measurements. ${ }^{28}$ It is noteworthy that irrespective of BTMs being associated with an individual's fracture risk, such markers are currently not included in any available fracture risk assessment tool. Possible reasons are the considerable diurnal variability of such markers and the fact that their significance in men is less well investigated compared with women. ${ }^{27}$ As a result, bone resorption markers including CTx and TRAP, as well as bone formation markers including OC, PINP and bALP will be measured in this study. A modelling algorithm (to be discussed later) will be applied to reduce the preanalytical variability.

\section{Genetic variability}

Recently, research activities concerning alternative circulating markers that could provide information on a person's fracture probability, such as SNPs have increased. In one of the largest meta-analyses performed on SNPs so far, it was found that about 56 gene loci are associated with BMD; of those about 14 are linked with fracture risk. ${ }^{29}{ }^{30}$ Despite these findings, these markers have never been integrated into any of the available assessment tools, possibly due to their modest effect size on fracture risk (ORs ranging from 1.03 to 1.10) ${ }^{31}$ Detecting SNPs that will later be used in the fracture risk assessment tool, therefore, is an integral part of the prevailing project.

\section{PROPOSED STATISTICAL METHODS}

Quantitative data will be described as mean \pm SD for normally distributed data, based on the Shapiro-Wilk test, while non-normal variables will be presented as median (IQR: P25-P75). Categorical data will be explained by percentages and will be tested using $\chi^{2}$, Fisher's exact. Between-group differences will be analysed using one-way analysis of variance, Student's t-test, Kruskal-Wallis and Mann-Whitney U tests, where indicated.

Incidence rates of osteoporosis and sarcopenia will be estimated per 1000 person-years. Also, relative risk, OR and HR with $95 \%$ CIs will be calculated to show the association of risk factors of interest with the outcomes. In the modelling of hip fracture, penalised methods will be used to alleviate probable overfitting due to possible small number of events per parameters. The optimism and the optimism-corrected performance will be calculated using bootstrap resampling methods to prevent overfitting. As cohorts can be considered as the strata, k-fold cross-validation techniques will be applied to compare the results.

Pearson's and Spearman's correlation coefficients will be used to present the correlation between continuous variables. Multiple linear regression analysis will be used to assess the association of continuous dependent variables with their potential risk factors. In the case of a dichotomous outcome, binary logistic regression or modified Poisson will be used.

To analyse the survival rates, the life table and KaplanMeier method will be used. Cox-proportional hazard models or other appropriate multivariable survival methods will be used to adjust for potential confounders.

As it is inherent in any cohort design, different correlations might be extracted from the studied population. It is therefore routine to use sex and age as covariates, as long as they are not separately correlated with the outcome of desire. However, other factors such as cognitive status may also be considered as covariates in more complex models. Choosing covariates will be carried out following the Hosmer-Lemeshow guidelines and referencing the previous body of evidence. 


\section{Device accuracy}

As for the PoCOsteo device, the agreement between the results measured using PoCOsteo and the gold standard methods (ECLIA and PCR) will be studied. Sensitivity, specificity and the $95 \%$ CIs for all comparisons will be calculated; these measures may need to be stratified based on factors such as gender or age. To assess the agreement between standard methods and results of this study, the Bland-Altman graph and Cohen's kappa coefficient $(\kappa)$ will be applied. A receiver operating characteristic analysis will be conducted to provide insight on optimal thresholds for PoCOsteo measurements. The curves, their coordinates and diagnostic measures will be reported.

Missing values will be replaced using multiple imputations through multiple regression method. In case of death, the data that should have been collected in the subsequent time points, will be censored at the final visit point.

\section{Modeling plans}

To provide a holistic statistical model that compensates for the preclinical variability of BTMs, variables such as age, previous medical and fracture history, and lifestyle measures (such as physical activity, dairy intake and alcohol consumption) along with BTMs and serum calcium and 25-hydroxyvitamin D levels will be used.

First, the correlation between each of the aforementioned variables with each marker will be assessed. This is followed by the application of linear regression models to study the correlation direction between the variables and its effect size. The final goal will be to compensate for the preclinical variability of BTMs.

A second modelling will be built to predict fracture risk using biomarker levels, genetic variability and CRFs, and illustrate a better outlook on how well using BTMs will assist clinical detection of high-risk patients. In this regard, data mining methods such as logistic regression, generalised boosted models, Artificial Neural Network and Super learner and Swiss Cheese Model will be applied.

Since overfitting is a central problem in prediction modelling, the validity of the model will be tested in new patients. In this regard, the ability of the device in predicting osteoporotic fracture will be tested after integrating this equation model into the PoCOsteo tool. At this point, DXA will serve as the gold standard for diagnosing osteoporosis. In other words, in the modelling phase, the existing dataset will be used as the training set and will be validated using those collected in this study.

\section{Sample size}

The prevalence of osteoporosis in BEH Cohort is about $28.8 \%$ and the overall prevalence of osteoporosis in Iran is reported to be $15 \%$. Based on the amount of squared multiple correlations between predictors and considering the attrition rate of about 10\%, 1628 individuals are needed for the Iranian part. However, considering the population-based nature of this arm, a total sample size of 2000 individuals including 500 osteoporotic and 1500 healthy participants will be needed.

As for Austria, the prevalence of osteoporosis is about $8 \%$. At the same time, the risk of experiencing and not experiencing fracture is $1.15 \%$ and $0.38 \%$, respectively. Considering the attrition rate of about $10 \%, 1131$ subjects are needed.

\section{ETHICS AND DISSEMINATION}

These studies are conducted in accordance with the World Medical Association Declaration of Helsinki. The Iranian part was approved by the Research Ethics Committee of EMRI, Tehran University of Medical Sciences, (IR.TUMS.EMRI.REC.1394.0036, IR.TUMS. EMRI.REC.1395.00152). The Austrian part was approved by the Ethical Board Committee of Graz University of Medical Sciences (30-246 ex 17/18).

As the study is granted by the H2020 projects, the partners will follow European Commission regulations for publication in open access journals and data management.

\section{Author affiliations}

${ }^{1}$ Center for Microsystems Technology, Imec \& Ghent University, Zwijnaarde - Gent, Belgium

${ }^{2}$ Department of Internal Medicine, Division of Endocrinology and Diabetology, Medical University of Graz, Graz, Steiermark, Austria

${ }^{3}$ Osteoporosis Research Center, Endocrinology and Metabolism Clinical Sciences Institute, Tehran University of Medical Sciences, Tehran, Iran (the Islamic Republic of)

${ }^{4}$ Department of Epidemiology and Biostatistics, Pasteur Institute of Iran, Tehran, Iran (the Islamic Republic of)

${ }^{5}$ The Persian Gulf Marine Biotechnology Research Center, The Persian Gulf Biomedical Sciences Research Institute, Bushehr University of Medical Sciences, Bushehr, Iran (the Islamic Republic of)

${ }^{6}$ Endocrinology and Metabolism Research Center, Endocrinology and Metabolism Clinical Sciences Institute, Tehran University of Medical Sciences, Tehran, Iran (the Islamic Republic of)

Contributors $\mathrm{PK}, \mathrm{HPD}, \mathrm{NM}, \mathrm{NF}$ and $\mathrm{AO}$ were involved in designing and drafting the PoCOsteo protocol and then characterising it for the Iranian and Austrian part separately. HPD, IN and BL supervised the process. PK and NM wrote the manuscript. SG drafted the statistical section. AO, IN, BL and HPD reviewed the manuscript.

Funding PoCOsteo is funded by a grant from HORIZON 2020 under contract number 767325. BEH Programme is jointly funded by the Endocrinology and Metabolism Research Institute affiliated to Tehran University of Medical Sciences and the Persian Gulf Biomedical Sciences Research Institute affiliated to Bushehr University of Medical Sciences.

Competing interests None declared.

Patient and public involvement Patients and/or the public were not involved in the design, or conduct, or reporting, or dissemination plans of this research.

Patient consent for publication Not required.

Provenance and peer review Not commissioned; externally peer reviewed.

Open access This is an open access article distributed in accordance with the Creative Commons Attribution Non Commercial (CC BY-NC 4.0) license, which permits others to distribute, remix, adapt, build upon this work non-commercially, and license their derivative works on different terms, provided the original work is properly cited, appropriate credit is given, any changes made indicated, and the use is non-commercial. See: http://creativecommons.org/licenses/by-nc/4.0/.

ORCID iD

Bagher Larijani http://orcid.org/0000-0001-5386-7597 


\section{REFERENCES}

1 World Health Organization. WHO | Facts about ageing [Internet]. WHO. World Health Organization, 2015. Available: https://www.who. int/ageing/about/facts/en/ [Accessed cited 2019 Feb 18].

2 Labarthe DR, Dunbar SB. Global cardiovascular health promotion and disease prevention: 2011 and beyond. Circulation 2012;125:2667-76.

3 Shetty P. Grey matter: ageing in developing countries. Lancet 2012;379:1285-7.

4 Weintraub WS, Daniels SR, Burke LE, et al. Value of primordial and primary prevention for cardiovascular disease. Circulation 2011;124:967-90.

5 Vijayakumar R, Büsselberg D. Osteoporosis: an under-recognized public health problem local and global risk factors and its regional and worldwide prevalence. J Local Glob Heal Sci 2016;2.

6 GBD 2015 SDG Collaborators. Measuring the health-related sustainable development goals in 188 countries: a baseline analysis from the global burden of disease study 2015. Lancet 2016;388:1813-50.

7 Kanis JA, Adachi JD, Cooper C, et al. Standardising the descriptive epidemiology of osteoporosis: recommendations from the epidemiology and quality of life Working group of IOF. Osteoporos Int 2013;24:2763-4.

8 Kanis JA, Svedbom A, Harvey N, et al. The osteoporosis treatment gap. J Bone Miner Res 2014;29:1926-8.

9 El-Hajj Fuleihan G, Adib G, Nauroy L. Epidemiology, costs \& burden of osteoporosis in 2011. The Middle East \& Africa regional audit 2011.

10 Statistical Centre of Iran. Islamic Republic of Iran vice presidency for strategic planning and supervision statistical centre of Iran selected findings of national population and housing census, 2011. Tehran 2012.

11 Marshall D, Johnell O, Wedel H. Meta-Analysis of how well measures of bone mineral density predict occurrence of osteoporotic fractures. BMJ 1996;312:1254-9.

12 Nayak S, Roberts MS, Greenspan SL. Cost-Effectiveness of different screening strategies for osteoporosis in postmenopausal women. Ann Intern Med 2011;155:751.

13 Khashayar P, Meybodi HA, Amoabediny G, et al. Biochemical markers of bone turnover and their role in osteoporosis diagnosis: a narrative review. Recent Pat Endocr Metab Immune Drug Discov 2015;9:79-89.

14 Silverman SL, Calderon AD. The utility and limitations of FRAX: a US perspective. Curr Osteoporos Rep 2010;8:192-7.

15 Kanis JA, Oden A, Johansson $\mathrm{H}$, et al. FRAX and its applications to clinical practice. Bone 2009;44:734-43.

16 Westesson P-L, Lee RK, Ketkar MA, et al. Underdiagnosis and undertreatment of osteoporosis. Lancet 2002;360:1891.
17 Khashayar P, Amoabediny G, Larijani B, et al. A multiplexed microfluidic platform for bone marker measurement: a proof-ofconcept. Micromachines 2017;8:133.

18 Shafiee G, Ostovar A, Heshmat R, et al. Bushehr elderly health (BEH) programme: study protocol and design of musculoskeletal system and cognitive function (stage II). BMJ Open 2017;7:e013606.

19 Abolhassani F, Mohammadi M, Soltani A. Burden of osteoporosis in Iran. Iran J Publ Heal 2004:18-28.

20 Dorner T, Weichselbaum E, Lawrence K, et al. Austrian osteoporosis report: epidemiology, lifestyle factors, public health strategies. Wien Med Wochenschr 2009;159:221-9.

21 Chiba K, Okazaki N, Kurogi A, et al. Precision of second-generation high-resolution peripheral quantitative computed tomography: intra- and Intertester Reproducibilities and factors involved in the reproducibility of cortical porosity. J Clin Densitom 2018;21:295-302.

22 Dimai HP, Svedbom A, Fahrleitner-Pammer A, et al. Epidemiology of hip fractures in Austria: evidence for a change in the secular trend. Osteoporos Int [Internet] 2011;22:685-92 http://www.ncbi.nlm.nih. $\mathrm{gov} / \mathrm{pubmed} / 20458573$

23 Moayyeri A, Soltani A, Larijani B, et al. Epidemiology of hip fracture in Iran: results from the Iranian multicenter study on accidental injuries. Osteoporos Int 2006;17:1252-7.

24 Taguchi A. Panoramic radiographs for identifying individuals with undetected osteoporosis. Jpn Dent Sci Rev [Internet]. Elsevier 2009;45:109-20 https://www.sciencedirect.com/science/article/pii/ S1882761609000349

25 Ehsanbakhsh AR, Akhbari H, Iraee MB, et al. The prevalence of undetected vertebral fracture in patients with back pain by dualenergy X-ray absorptiometry (DXA) of the lateral thoracic and lumbar spine. Asian Spine J 2011;5:139-45.

26 Dimai HP. Use of dual-energy X-ray absorptiometry (DXA) for diagnosis and fracture risk assessment; WHO-criteria, T- and Zscore, and reference databases. Bone 2017;104:39-43.

27 Vilaca T, Gossiel F, Eastell R. Bone turnover markers: use in fracture prediction. J Clin Densitom 2017;20:346-52.

28 Vasikaran S, Eastell R, Bruyère $\mathrm{O}$, et al. Markers of bone turnover for the prediction of fracture risk and monitoring of osteoporosis treatment: a need for international reference standards. Osteoporos Int 2011;22:391-420.

29 Estrada K, Styrkarsdottir U, Evangelou E, et al. Genome-Wide metaanalysis identifies 56 bone mineral density loci and reveals 14 loci associated with risk of fracture. Nat Genet 2012:44:491-501.

30 Medina-Gomez C, Kemp JP, Trajanoska K, et al. Life-Course genome-wide association study meta-analysis of total body BMD and assessment of age-specific effects. Am J Hum Genet 2018;102:88-102.

31 Trajanoska K, Morris JA, Oei L, et al. Assessment of the genetic and clinical determinants of fracture risk: genome wide association and Mendelian randomisation study. BMJ 2018;362:k3225. 